University of Warwick institutional repository: http://go.warwick.ac.uk/wrap This paper is made available online in accordance with publisher policies. Please scroll down to view the document itself. Please refer to the repository record for this item and our policy information available from the repository home page for further information.

To see the final version of this paper please visit the publisher's website. Access to the published version may require a subscription.

Author(s): E. Eirian JONES, Alison STEWART and John M. WHIPPS Article Title: Use of Coniothyrium minitans transformed with the hygromycin B resistance gene to study survival and infection of Sclerotinia sclerotiorum sclerotia in soil Year of publication: 2003 Link to published version: http://dx.doi.org/10.1017/S0953756203007457 Publisher statement: None 


\title{
Use of Coniothyrium minitans transformed with the hygromycin B resistance gene to study survival and infection of Sclerotinia sclerotiorum sclerotia in soil
}

\author{
E. Eirian JONES ${ }^{1 *}$, Alison STEWART ${ }^{2}$ and John M. WHIPPS ${ }^{1}$ \\ ${ }^{1}$ Horticulture Research International, Wellesbourne, Warwick, CV35 9EF, UK. \\ ${ }^{2}$ Soil, Plant and Ecological Sciences Division, PO Box 84, Lincoln University, New Zealand. \\ E-mail:Eirian.Jones@hri.ac.uk
}

Received 7 August 2002; accepted 18 January 2003.

\begin{abstract}
A Coniothyrium minitans strain (T3) co-transformed with the genes for $\beta$-glucuronidase (uidA) and hygromycin phosphotransferase $(h p h)$, the latter providing resistance to the antibiotic hygromycin $\mathrm{B}$, was used to investigate the survival and infection of sclerotia of Sclerotinia sclerotiorum by C. minitans over time in four different soils. Infection of sclerotia was rapid in all cases, with the behaviour of transformant T3 and wild type parent A69 being similar. Differences were seen between the soils in the rate of infection of sclerotia by $C$. minitans and in their indigenous fungal populations. Amendment of agar with hygromycin B enabled the quantification of $C$. minitans in soil by dilution plating where there was a high background of other microorganisms. In Lincoln soil from New Zealand, which had a natural but low population of $C$. minitans, the hygromycin B resistance marker allowed the umambiguous discrimination of the applied transformed isolate from the indigenous hygromycin B sensitive one. In this soil, although the indigenous C. minitans population was detected from sclerotia, none were recovered on the dilution plates, indicating the increased sensitivity of $C$. minitans detection from soil using sclerotial baiting. $C$. minitans was a very efficient parasite, being able to infect a large proportion of sclerotia within a relatively short time from an initially low soil population. The addition of hygromycin B to agar also allowed the detection of $C$. minitans from decaying sclerotia by inhibiting secondary fungal colonisers. This is the first report to show that fungi colonising sclerotia already infected by $C$. minitans mask the detection of $C$. minitans from sclerotia rather than displacing the original parasite.
\end{abstract}

\section{INTRODUCTION}

The sclerotial mycoparasite Coniothyrium minitans is a well-documented biocontrol agent of the widespread soil-borne plant pathogen Sclerotinia sclerotiorum. It infects and degrades the sclerotia of S. sclerotiorum in soil (Trutmann, Keane \& Merriman 1980, McQuilken \& Whipps 1995, Jones \& Whipps 2002) and has been shown to control the pathogen in both field and glasshouse trials (Whipps, Budge \& Ebben 1989, McLaren et al. 1994, McQuilken \& Whipps 1995, Gerlagh et al. 1999, Huang et al. 2000, Jones \& Whipps 2002). However, information about the ecology of $C$. minitans in soil in relation to sclerotia is limited (Whipps \& Gerlagh 1992). Its presence in soil is often indicated by its recovery from infected sclerotia (Huang 1981, SandysWinsch et al. 1993) although previous reports have suggested that it can be displaced from sclerotia by secondary colonisers (McCredie \& Sivasithamparam 1985, Jones \& Stewart 2000). Additionally, it is often outcompeted on soil dilution plates by fast-growing fungi

* Corresponding author. resulting in an under estimation of its population in soil, and attempts at developing a selection medium have been unsuccessful (Williams 1996). Currently, it is detected from soils using media containing antibiotics and Triton $X$ to slow other fungi (Whipps et al. 1989) but this does not completely alleviate the problem of plate competition. Although there are two commercial C. minitans products, Contans ${ }^{\circledR}$ and KONI (Whipps \& Davies 2000), a better understanding of the ecology of C. minitans and its interrelationship with S. sclerotior$u m$ in soil could help to target the biocontrol agent to maximise its effect.

A key requirement to enable the monitoring of the survival and spread of $C$. minitans in soil is the ability to detect unambiguously and monitor the specific isolate applied. One such means is by constitutive expression of reporter genes or other selectable antibiotic resistance genes. Jones et al. (1999) co-transformed C. minitans isolate A69 with the genes encoding $\beta$ glucuronidase (uidA) and hygromycin phosphotransferase $(h p h)$. The uidA gene has been used by other workers to quantify biomass of Cladosporium fulvum (Oliver et al. 1993), to measure metabolic activity of 
Trichoderma harzianum (Green \& Funck Jensen 1995, Green et al. 1999), to monitor root colonisation by Fusarium oxysporum (Eparvier \& Alabouvette 1994) and to examine root infection by Bipolaris sorokiniana (Liljeroth, Jansson \& Schäfer 1993). The hygromycin phosphotransferase gene allows the selective isolation and quantification of $C$. minitans from soil samples on media containing hygromycin B enabling the survival and colonisation of sclerotia and other substrates by C. minitans over time to be monitored. In an initial study, two transformants, T3 and T4, were found to be the most similar in a range of biological characteristics to the wild-type isolate A69 (parent) (Jones et al. 1999). In this study, transformant T3 was used to investigate the survival of $C$. minitans and its infection of sclerotia over time in four different soils utilising the hygromycin $\mathrm{B}$ resistance characteristic.

\section{MATERIALS AND METHODS}

\section{Source and maintenance of fungi}

Coniothyrium minitans isolate A69 was isolated from sclerotia of Sclerotium cepivorum obtained from Pukekohe, NZ (Jones \& Stewart 2000) with transformant T3 being a uidA and hph transformant of A69 (Jones et al. 1999). Sclerotinia sclerotiorum isolate G18 was isolated from diseased carrot, Christchurch, NZ (Jones \& Stewart 2000). The fungi were stored in polypropylene straw ampoules in liquid $\mathrm{N}_{2}$ (Challen \& Elliott 1986) and routinely cultured at $18-20{ }^{\circ} \mathrm{C}$ on potato dextrose agar (PDA; Oxoid, Basingstoke). All strains are maintained in the culture collection of Horticulture Research International (HRI), Wellesbourne.

\section{Sclerotial production}

Sclerotia of Sclerotinia sclerotiorum isolate G18 were produced using the method of Mylchreest \& Wheeler (1987). $25 \mathrm{~g}$ of wheat grain (cv. 'Armada') and $60 \mathrm{ml}$ distilled water were autoclaved and, once cooled, were inoculated with three discs $(10 \mathrm{~mm}$ in diameter $)$ cut from the colony margin of 2-3 d old PDA cultures of S. sclerotiorum. Flasks were incubated at $20^{\circ}$ for $4 \mathrm{wk}$ in darkness with the flasks shaken after $1 \mathrm{wk}$ to facilitate mixing of the inoculum. The sclerotia were then washed, dried overnight in a stream of sterile air, and those of 2-4 mm diam were selected and used immediately.

\section{Production of maizemeal-perlite inoculum of C. minitans}

Inocula of Coniothyrium minitans A69 (wild-type) and transformant T3 were produced on maizemeal-perlite. $100 \mathrm{~cm}^{3}$ of a 15:85\% (v:v) maizemeal (Midlands Shire Farmers, Worcester) to horticultural grade perlite (Silvaperl Products, Harrogate) mixture was placed in a
$250 \mathrm{ml}$ conical flask and moistened with $20 \mathrm{ml}$ tap water and autoclaved. After cooling, each flask was inoculated with $5 \mathrm{ml}$ of a suspension containing $1 \times 10^{6}$ conidia $\mathrm{ml}^{-1}$ produced from $14 \mathrm{~d}$ old PDA cultures of either C. minitans isolate A69 or transformant $\mathrm{T} 3$, and incubated at $20^{\circ}$ in the dark for $4 \mathrm{wk}$. The flasks were shaken once a week to facilitate mixing of inoculum.

\section{Coniothyrium minitans sclerotial parasitism}

Parasitism of Sclerotinia sclerotiorum sclerotia by Coniothyrium minitans in soil was investigated using a modification of the method of Jones et al. (1999). Four soils were used: Kirton, Lincolnshire, UK (clay loam; Romney series); Lincoln, Canterbury, NZ (silt loam, Wakanui series); Rixton, Cheshire, UK (peat; Turbary Moor series) and Wellesbourne, Warwicks, UK (loam clay; Dunnington Heath series).

For each soil type, $10 \mathrm{~cm}^{3}$ of $C$. minitans A69 or transformant T3 colonised maizemeal-perlite was ground using a mortar and pestle and mixed thoroughly with $990 \mathrm{~cm}^{3}$ of air-dried soil (sieved $4 \mathrm{~mm}$ ). A quantity of this mixture (30 $\mathrm{g}$ for Rixton soil which has a low bulk density, and $50 \mathrm{~g}$ for the other three mineral soils) was placed in each of 15 Petri dishes and moistened to $-0.1 \mathrm{MPa}(18.5 \mathrm{ml}$ per $30 \mathrm{~g}$ Rixton soil and $9.8 \mathrm{ml}$, $9.0 \mathrm{ml}$ and $13.6 \mathrm{ml}$ per $50 \mathrm{~g}$ soil for Kirton, Lincoln and Wellesbourne soils, respectively) with sterile distilled water (SDW). For each treatment, 20 sclerotia (2-4 mm diam) were pressed lightly into the surface of the inoculated soil in each of 15 Petri dishes and incubated at $20^{\circ}$ in the dark. The water content of plates was adjusted to $-0.1 \mathrm{MPa}$ weekly. Sclerotia placed on noninoculated soil were used as a comparative control. After 1, 2, 4, 6 and $8 \mathrm{wk}$, three plates per treatment were randomly selected and the sclerotia harvested and surface sterilised by agitation in $50 \mathrm{ml}$ of a $50: 50$ (v:v) solution of 13-15\% sodium hypochlorite (Hays Chemical distribution, Birmingham) and absolute ethanol for $3 \mathrm{~min}$ (Jones et al. 2003). This was followed by rinsing individual sclerotia twice for $1 \mathrm{~min}$ in $2 \mathrm{ml} \mathrm{SDW}$ in separate wells of a 25 well Petri dish (Bibby Sterilin, Stone). Washing the sclerotia individually eliminated possible cross contamination of sclerotia by a heavily infected sclerotium that could occur during batch washing of sclerotia (Budge \& Whipps 2001). The sclerotia were bisected and placed on pairs of agar discs (15 mm diam) in a Petri dish. One of each agar disc pair consisted of PDA supplemented with chlortetracycline $\left(20 \mu \mathrm{g}^{-1}\right.$ powder containing $80 \%$ chlortetracycline $\mathrm{HCl}$; Sigma Chemicals, Poole) while the other consisted of PDA supplemented with chlortetracycline $\left(20 \mu \mathrm{g}^{-1}\right)$ and hygromycin B (80 $\mu \mathrm{g}^{-\mathbf{1}}$; Boehringer Mannheim Biochemicals, Mannheim). Discs were incubated at $20^{\circ}$ for $10 \mathrm{~d}$ in the dark and assessed for the number of sclerotia showing mycelial growth of $S$. sclerotiorum (viable) and/or infection by $C$. minitans. 


\section{Coniothyrium minitans survival in soil}

Colony forming unit (cfu) counts for the maizemealperlite inocula were estimated using standard dilution plating techniques (Jones et al. 1999) whereby $10 \mathrm{~cm}^{3}$ of maizemeal-perlite inocula were shaken in $90 \mathrm{ml}$ of $0.01 \%(\mathrm{w} / \mathrm{v})$ sterile technical agar No. 3 (Oxoid) for $10 \mathrm{~min}$. After standing for a further $20 \mathrm{~min}$, serial dilutions were made and plated on three plates of both Czapex Dox agar (CDA; Oxoid) supplemented with chlortetracycline $\left(20 \mu \mathrm{g}^{-1}\right)$ and Triton X-100 ( $2 \mathrm{mll}^{-1}$; Sigma Chemicals) and CDA supplemented with chlortetracycline $\left(20 \mu \mathrm{g} \mathrm{1^{-1 }}\right)$ and hygromycin B $\left(80 \mu \mathrm{g}^{-1}\right)$. Recovery and survival of $C$. minitans in the soil at the start of the assay and at each harvest was assessed by soil dilution. A $10 \mathrm{~cm}^{3}$ soil sample for each treatment was diluted with $90 \mathrm{ml}$ sterile $0.01 \%$ agar, and treated as described previously.

\section{Design and statistical analysis}

The treatments were arranged as a randomised design with three replicates of 20 sclerotia per treatment. All percentage data were arcsine transformed, to satisfy the assumption of homogeneity of variance, prior to analysis using analysis of variance (ANOVA) in GenStat for Windows (Payne 2000) assuming a completely randomised design. Percentage infection of sclerotia with $C$. minitans and percentage other fungi were analysed for all combinations of treatment, agar $(+/-$ hygromycin B) and week. As many of the observations were zero, the residual mean squares from these analyses were likely to be deflated, thus inflating the significance level for treatment comparisons, and so comparisons were only noted at the $1 \%$ significance level. As S. sclerotiorum was only able to grow on the unamended discs, observations on hygromycin Bamended agar discs were omitted from the analysis of percentage sclerotial viability, and comparisons were noted at the $5 \%$ significance level.

Colony forming unit data were $\log _{10}$ transformed, to satisfy the assumption of homogeneity of variance, prior to analysis using ANOVA assuming a completely randomised design.

\section{RESULTS}

\section{Coniothyrium minitans sclerotial parasitism}

For all soils, viability of sclerotia incubated in soils treated with Coniothyrium minitans isolate A69 or transformant T3 treated soils significantly decreased with incubation time (Tables 1-4). Additionally, for both C. minitans treatments, the percentage of sclerotia infected with $C$. minitans increased to a maximum after 2-4 wk on media lacking hygromycin B. With increased incubation ( $8 \mathrm{wk}$ ), recovery of $C$. minitans from sclerotia significantly decreased. In general, at the later harvest times $(4,6$ and $8 \mathrm{wk})$, percentage recovery of
C. minitans from sclerotia incubated in transformant T3 treated soils was significantly higher on hygromycin B-amended agar compared with agar lacking hygromycin B. Concurrently, recovery of fungi other than C. minitans was generally significantly decreased on hygromycin B-amended agar compared with unamended agar from soils treated with either isolate A69 or transformant T3 at these harvest times. Nevertheless, there were some additional significant differences in viability and infection of sclerotia between the various soils and these are detailed below.

In both untreated Rixton and Wellesbourne soils, the majority of the sclerotia recovered were viable throughout the experiment (over 90\%) (Tables 3-4). However, for both uninoculated Kirton and Lincoln soils, although sclerotial viability was initially high, viability significantly decreased with increased incubation (Tables 1-2). Concurrently, for sclerotia incubated in uninoculated Lincoln soil, C. minitans was initially recovered at a low level $(0.6 \%$ infection at wk 1$)$, but significantly increased with incubation. No C. minitans was isolated from any sclerotia incubated in any of the other three uninoculated soils.

In soils treated with isolate A69 or transformant T3 treated soil, recovery of $C$. minitans from sclerotia harvested after $8 \mathrm{wk}$ incubation was low for all soils (less than 12\%), apart from Rixton soil where over $40 \%$ of sclerotia were infected by C. minitans (Tables 1-4). Infection of sclerotia with fungi other than $C$. minitans also varied in the four soils. For uninoculated Kirton, Rixton and Wellesbourne soils, infection of sclerotia with fungi other than $C$. minitans generally increased with incubation. However, for sclerotia incubated in the uninoculated Lincoln soil, infection with fungi other than C. minitans was low throughout, with no significant difference between the numbers recovered at any incubation time. Infection of sclerotia incubated in Rixton soil treated with isolate A69 or transformant T3 by fungi other than C. minitans was low throughout. However, infection of sclerotia with fungi other than C. minitans recovered from Lincoln, Rixton and Wellesbourne soils treated with isolate A69 or transformant T3 was high, with the maximum recovery at the $6 \mathrm{wk}$ harvest $(31.3,32.2$ and $33.3 \%$, respectively for Kirton, Lincoln and Wellesbourne soils treated with isolate A69, and 28.0, 55.5 and $51.7 \%$, respectively for treated Kirton, Lincoln and Wellesbourne soils treated with transformant T3).

For all four soils, no C. minitans colonies were isolated on hygromycin B-amended agar from treatments amended with isolate A69. Similarly the native C. minitans recovered from uninoculated Lincoln soil was also found to be sensitive to hygromycin B.

\section{Coniothyrium minitans survival in soil}

Inoculum levels in maizemeal-perlite inoculum for isolate A69 and transformant T3 were 7.3-7.7 $\log _{10}$ cfu $\mathrm{cm}^{-3}$ maizemeal-perlite on CDA amended with 
Table 1. Kirton soil: Viability (V) and infection of Sclerotinia sclerotiorum sclerotia by Coniothyrium minitans (Cm) or other fungi (other) incubated in Kirton soil amended with C. minitans isolate A69 (wild-type) or transformant T3 over time. Mean of three replicate plates of 20 sclerotia per incubation time per treatment. Values in parentheses are means after arcsin transformations of percentage data.

\begin{tabular}{|c|c|c|c|c|c|c|c|c|c|c|}
\hline \multirow[b]{3}{*}{ Treatments } & \multicolumn{10}{|c|}{ Percentage of sclerotia viable or infected with $C$. minitans or other fungi } \\
\hline & \multicolumn{2}{|l|}{$1 \mathrm{wk}$} & \multicolumn{2}{|l|}{$2 \mathrm{wk}$} & \multicolumn{2}{|l|}{$4 \mathrm{wk}$} & \multicolumn{2}{|l|}{$6 \mathrm{wk}$} & \multicolumn{2}{|l|}{$8 \mathrm{wk}$} \\
\hline & - hyg $^{\text {a }}$ & + hyg $^{\mathrm{b}}$ & - hyg & +hyg & - hyg & +hyg & - hyg & +hyg & - hyg & + hyg \\
\hline \multicolumn{11}{|c|}{ Untreated control } \\
\hline V & $100(90.0)$ & $\mathrm{NC}^{\mathrm{c}}$ & $99.4(85.7)$ & $\mathrm{NC}$ & $97.8(81.4)$ & NC & $87.8(69.6)$ & $\mathrm{NC}$ & $79.0(62.7)$ & $\mathrm{NC}$ \\
\hline $\mathrm{Cm}^{\mathrm{d}}$ & $0(0)$ & $0(0)$ & $0(0)$ & $0(0)$ & $0(0)$ & $0(0)$ & $0(0)$ & $0(0)$ & $0(0)$ & $0(0)$ \\
\hline Other & $0(0)$ & $0(0)$ & $4.5(12.3)$ & $0(0)$ & $3.3(10.5)$ & $0(0)$ & $13.5(21.5)$ & $0(0)$ & $26.0(30.7)$ & $0(0)$ \\
\hline \multicolumn{11}{|c|}{ C. minitans A69 } \\
\hline V & $63.9(53.1)$ & $\mathrm{NC}$ & $24.9(29.9)$ & $\mathrm{NC}$ & $9.8(18.2)$ & $\mathrm{NC}$ & $14.1(22.0)$ & $\mathrm{NC}$ & $9.6(18.1)$ & $\mathrm{NC}$ \\
\hline $\mathrm{Cm}$ & $58.4(49.8)$ & $0(0)$ & $73.8(59.2)$ & $0(0)$ & $69.9(56.7)$ & $0(0)$ & $15.2(23.0)$ & $0(0)$ & $5.6(13.7)$ & $0(0)$ \\
\hline Other & $3.3(10.5)$ & $0(0)$ & $7.8(16.2)$ & $0(0)$ & $15.4(23.1)$ & $0(0)$ & $31.3(34.0)$ & $0(0)$ & $21.6(27.7)$ & $0(0)$ \\
\hline \multicolumn{11}{|c|}{ C. minitans $\mathrm{T} 3$} \\
\hline V & $62.0(51.9)$ & $\mathrm{NC}$ & $8.2(16.6)$ & $\mathrm{NC}$ & $7.8(16.2)$ & $\mathrm{NC}$ & $10.0(18.4)$ & $\mathrm{NC}$ & $0.6(4.3)$ & $\mathrm{NC}$ \\
\hline $\mathrm{Cm}$ & $65.5(54.1)$ & $70.5(57.1)$ & $97.8(81.4)$ & $94.4(76.3)$ & $62.0(51.9)$ & $82.1(65.0)$ & $8.0(17.2)$ & $32.8(34.9)$ & $2.2(8.6)$ & $12.2(20.5)$ \\
\hline Other & $0(0)$ & $0(0)$ & $4.3(11.9)$ & $0(0)$ & $20.4(26.8)$ & $0(0)$ & $28.0(31.9)$ & $0.6(4.3)$ & $10.2(18.6)$ & $0(0)$ \\
\hline $\begin{array}{l}\text { LSD }(5 \%, 3 \\
\text { LSD }(1 \%, 6 \\
\text { LSD }(1 \%, 6\end{array}$ & $\operatorname{LSD}\left(1 \%, 60\right.$ d.f.) $\mathrm{Cm}$ (transformed data) $=14.96^{\mathrm{f}}$ & $\begin{array}{l}3.05^{\mathrm{e}} \\
=14.96^{\mathrm{f}} \\
=14.96^{\mathrm{g}}\end{array}$ & & & & & & & & \\
\hline
\end{tabular}

${ }^{\text {a }}$ PDA with chlortetracycline; ${ }^{\mathrm{b}}$ PDA with hygromycin B and chlortetracycline; ${ }^{\mathrm{c}}$ Not applicable; ${ }^{\mathrm{d}} \mathrm{Cm}$ data for the - hyg plates can include any $C$. minitans strain; however for the + hyg plates, only $\mathrm{T} 3$ can be detected.

${ }^{\mathrm{e}}$ LSD for comparison of S. sclerotiorum recovery across weeks or between treatments. Significant differences between any treatment means were calculated from the least significant difference (LSD), where

$\mathrm{LSD}=t_{v} \times \mathrm{SED}$, and SED $=$ standard error of the difference between the means derived from ANOVA analysis and $t=$ critical value $(P=0.05)$ of Student's $t$ distribution for $v$ degrees of freedom (d.f.).

${ }^{\mathrm{f}}$ LSD for comparison of $C$. minitans recovery across weeks, between treatments or between amended and unamended hygromycin $\mathrm{B}$ discs at $(P=0.01)$.

$\mathrm{g}$ LSD for comparison of other fungi recovery across weeks, between treatments or between amended and unamended hygromycin $\mathrm{B}$ discs at $(P=0.01)$. 
Table 2. Lincoln soil: Viability (V) and infection of Sclerotinia sclerotiorum sclerotia by Coniothyrium minitans (Cm) or other fungi (other) incubated in Lincoln soil amended with C. minitans isolate A69 (wild-type) or transformant T3 over time. Mean of three replicate plates of 20 sclerotia per incubation time per treatment. Values in parentheses are means after arcsin transformations of percentage data.

\begin{tabular}{|c|c|c|c|c|c|c|c|c|c|c|}
\hline \multirow[b]{3}{*}{ Treatments } & \multicolumn{10}{|c|}{ Percentage of sclerotia viable or infected with C. minitans or other fungi } \\
\hline & \multicolumn{2}{|l|}{$1 \mathrm{wk}$} & \multicolumn{2}{|l|}{$2 \mathrm{wk}$} & \multicolumn{2}{|l|}{$4 \mathrm{wk}$} & \multicolumn{2}{|l|}{$6 \mathrm{wk}$} & \multicolumn{2}{|l|}{8 wk } \\
\hline & - hyg $^{\mathrm{a}}$ & + hyg $^{\mathrm{b}}$ & - hyg & + hyg & - hyg & + hyg & - hyg & + hyg & - hyg & + hyg \\
\hline \multicolumn{11}{|c|}{ Untreated control } \\
\hline $\mathrm{V}$ & $100(90)$ & $\mathrm{NC}^{\mathrm{c}}$ & $93.5(75.2)$ & $\mathrm{NC}$ & $83.5(66.0)$ & $\mathrm{NC}$ & $65.1(53.8)$ & $\mathrm{NC}$ & $75.4(60.3)$ & $\mathrm{NC}$ \\
\hline $\mathrm{Cm}^{\mathrm{d}}$ & $0.6(4.3)$ & $0(0)$ & $8.2(16.6)$ & $0(0)$ & $28.0(31.9)$ & $0(0)$ & $82.1(65.0)$ & $0(0)$ & $78.4(62.3)$ & $0(0)$ \\
\hline Other & $0.6(4.3)$ & $0(0)$ & $1.2(6.1)$ & $0.6(4.3)$ & $3.3(10.5)$ & $0.6(4.3)$ & $6.5(14.8)$ & $0(0)$ & $0.6(4.3)$ & $2.2(18.6)$ \\
\hline \multicolumn{11}{|c|}{ C. minitans $\mathrm{A} 69$} \\
\hline V & $79.1(62.8)$ & $\mathrm{NC}$ & $40.5(39.5)$ & $\mathrm{NC}$ & $16.9(24.3)$ & $\mathrm{NC}$ & $3.3(10.5)$ & $\mathrm{NC}$ & $6.9(15.2)$ & $\mathrm{NC}$ \\
\hline $\mathrm{Cm}$ & $51.8(46.0)$ & $0(0)$ & $65.2(53.9)$ & $0(0)$ & $95.8(78.1)$ & $0(0)$ & $65.5(54.1)$ & $0(0)$ & $0(0)$ & $0(0)$ \\
\hline Other & $0(0)$ & $0(0)$ & $2.2(8.6)$ & $0(0)$ & $4.3(11.9)$ & $0.6(4.3)$ & $32.3(34.6)$ & $9.0(17.5)$ & $23.2(28.8)$ & $0.6(4.3)$ \\
\hline \multicolumn{11}{|c|}{ C. minitans $\mathrm{T} 3$} \\
\hline V & $70.0(56.8)$ & $\mathrm{NC}$ & $26.4(31.0)$ & $\mathrm{NC}$ & $1.2(6.1)$ & $\mathrm{NC}$ & $1.2(6.1)$ & $\mathrm{NC}$ & $3.3(10.5)$ & $\mathrm{NC}$ \\
\hline $\mathrm{Cm}$ & $81.8(64.8)$ & $93.3(75.0)$ & $96.7(79.6)$ & $91.8(73.4)$ & $71.9(58.0)$ & $98.9(83.9)$ & $36.1(36.9)$ & $65.4(54.0)$ & $6.5(14.8)$ & $7.8(16.2)$ \\
\hline Other & $0.6(4.3)$ & $0(0)$ & $0.6(4.3)$ & $0(0)$ & $33.3(35.3)$ & $0.6(4.3)$ & $55.5(48.2)$ & $5.2(13.2)$ & $12.9(21.1)$ & $0(0)$ \\
\hline & \multicolumn{2}{|c|}{$\begin{array}{l}\operatorname{LSD}\left(5 \%, 30 \text { d.f.) } V(\text { transformed data })=14.70^{\mathrm{e}}\right. \\
\operatorname{LSD}\left(1 \%, 60 \text { d.f.) } \mathrm{Cm} \text { (transformed data) }=14.09^{\mathrm{f}}\right.\end{array}$} & & & & & & & & \\
\hline
\end{tabular}


Table 3. Rixton soil: Viability (V) and infection of Sclerotinia sclerotiorum sclerotia by Coniothyrium minitans $(\mathrm{Cm})$ or other fungi (other) incubated in Rixton soil amended with C. minitans isolate A69 (wild-type) or transformant T3 over time. Mean of three replicate plates of 20 sclerotia per incubation time per treatment. Values in parentheses are means after arcsin transformations of percentage data.

\begin{tabular}{|c|c|c|c|c|c|c|c|c|c|c|}
\hline \multirow[b]{3}{*}{ Treatments } & \multicolumn{10}{|c|}{ Percentage of sclerotia viable or infected with C. minitans or other fungi } \\
\hline & \multicolumn{2}{|l|}{$1 \mathrm{wk}$} & \multicolumn{2}{|l|}{$2 \mathrm{wk}$} & \multicolumn{2}{|l|}{$4 \mathrm{wk}$} & \multicolumn{2}{|l|}{$6 \mathrm{wk}$} & \multicolumn{2}{|l|}{$8 \mathrm{wk}$} \\
\hline & - hyg $^{\mathrm{a}}$ & + hyg $^{\mathrm{b}}$ & - hyg & +hyg & -hyg & + hyg & -hyg & + hyg & -hyg & +hyg \\
\hline \multicolumn{11}{|c|}{ Untreated control } \\
\hline $\mathrm{V}$ & $91.8(73.4)$ & $\mathrm{NC}^{\mathrm{c}}$ & $99.4(85.7)$ & $\mathrm{NC}$ & $97.8(81.4)$ & $\mathrm{NC}$ & $100(90.0)$ & $\mathrm{NC}$ & $100(90.0)$ & $\mathrm{NC}$ \\
\hline $\mathrm{Cm}^{\mathrm{d}}$ & $0(0)$ & $0(0)$ & $0(0)$ & $0(0)$ & $0(0)$ & $0(0)$ & $0(0)$ & $0(0)$ & $0(0)$ & $0(0)$ \\
\hline Other & $4.3(11.9)$ & $0(0)$ & $0.6(4.3)$ & $0(0)$ & $4.3(11.9)$ & $1.1(6.1)$ & $2.2(8.6)$ & $2.2(8.6)$ & $18.3(25.3)$ & $1.1(6.1)$ \\
\hline \multicolumn{11}{|c|}{ C. minitans A69 } \\
\hline $\mathrm{V}$ & $46.7(43.1)$ & $\mathrm{NC}$ & $23.0(28.7)$ & $\mathrm{NC}$ & $0.6(4.3)$ & $\mathrm{NC}$ & $2.2(8.6)$ & $\mathrm{NC}$ & $0(0)$ & $\mathrm{NC}$ \\
\hline $\mathrm{Cm}$ & $61.7(51.8)$ & $0(0)$ & $99.4(85.7)$ & $0(0)$ & $100(90)$ & $0(0)$ & $98.3(82.4)$ & $0(0)$ & $48.2(44.0)$ & $0(0)$ \\
\hline Other & $4.5(12.3)$ & $0.6(4.3)$ & $3.3(10.5)$ & $0(0)$ & $0(0)$ & $0(0)$ & $0(0)$ & $0(0)$ & $14.1(22.0)$ & $0(0)$ \\
\hline \multicolumn{11}{|c|}{ C. minitans $\mathrm{T} 3$} \\
\hline $\mathrm{V}$ & $46.7(43.1)$ & $\mathrm{NC}$ & $0.6(4.3)$ & $\mathrm{NC}$ & $0(0)$ & $\mathrm{NC}$ & $0(0)$ & $\mathrm{NC}$ & $0(0)$ & $\mathrm{NC}$ \\
\hline $\mathrm{Cm}$ & $77.0(61.3)$ & $95.8(78.1)$ & $97.8(81.4)$ & 99.4 (85.7) & $96.3(78.9)$ & $97.6(81.1)$ & $63.9(53.1)$ & $62.8(52.4)$ & $43.0(41.0)$ & $53.4(47.0)$ \\
\hline Other & $2.2(8.6)$ & $0(0)$ & $0(0)$ & $0(0)$ & $0(0)$ & $0(0)$ & $0(0)$ & $0(0)$ & $8.8(17.2)$ & $0(0)$ \\
\hline $\begin{array}{l}\operatorname{LSD}(5 \%, 3 \\
\operatorname{LSD}(1 \%, 6 \\
\operatorname{LSD}(1 \%, 6\end{array}$ & $\begin{array}{l}\text { ansformed d } \\
\text { (transformed } \\
\text { r (transform }\end{array}$ & $\begin{array}{l}=8.67^{\mathrm{e}} \\
=16.20^{\mathrm{f}} \\
\text { ta) }=14.37^{\mathrm{g}}\end{array}$ & & & & & & & & \\
\hline
\end{tabular}


Table 4. Wellesbourne soil: Viability (V) and infection of Sclerotinia sclerotiorum sclerotia by Coniothyrium minitans (Cm) or other fungi (other) incubated in Wellesbourne soil amended with C. minitans isolate A69 (wild-type) or transformant T3 over time. Mean of three replicate plates of 20 sclerotia per incubation time per treatment. Values in parentheses are means after arcsin transformations of percentage data.

\begin{tabular}{|c|c|c|c|c|c|c|c|c|c|c|}
\hline \multirow[b]{3}{*}{ Treatments } & \multicolumn{10}{|c|}{ Percentage of sclerotia viable or infected with C. minitans or other fungi } \\
\hline & \multicolumn{2}{|l|}{$1 \mathrm{wk}$} & \multicolumn{2}{|l|}{$2 \mathrm{wk}$} & \multicolumn{2}{|l|}{$4 \mathrm{wk}$} & \multicolumn{2}{|l|}{$6 \mathrm{wk}$} & \multicolumn{2}{|l|}{$8 \mathrm{wk}$} \\
\hline & - hyg $^{\mathrm{a}}$ & + hyg $^{\mathrm{b}}$ & - hyg & + hyg & - hyg & +hyg & - hyg & +hyg & -hyg & + hyg \\
\hline \multicolumn{11}{|c|}{ Untreated control } \\
\hline V & $100(90)$ & $\mathrm{NC}^{\mathrm{c}}$ & $99.4(85.7)$ & $\mathrm{NC}$ & $100(90.0)$ & $\mathrm{NC}$ & 99.4 (85.7) & $\mathrm{NC}$ & $93.5(75.2)$ & $\mathrm{NC}$ \\
\hline $\mathrm{Cm}^{\mathrm{d}}$ & $0(0)$ & $0(0)$ & $0(0)$ & $0(0)$ & $0(0)$ & $0(0)$ & $0(0)$ & $0(0)$ & $0(0)$ & $0(0)$ \\
\hline Other & $1.2(6.1)$ & $0.6(4.3)$ & $0.6(4.3)$ & $0(0)$ & $2.2(8.6)$ & $0(0)$ & $4.5(12.3)$ & $0(0)$ & $9.6(18.1)$ & $0(0)$ \\
\hline \multicolumn{11}{|c|}{ C. minitans A69 } \\
\hline V & 86.8 (68.7) & $\mathrm{NC}$ & $31.5(34.2)$ & $\mathrm{NC}$ & $21.1(27.4)$ & $\mathrm{NC}$ & $16.6(24.1)$ & $\mathrm{NC}$ & $11.6(19.9)$ & $\mathrm{NC}$ \\
\hline $\mathrm{Cm}$ & $48.3(44.0)$ & $0(0)$ & $65.5(54.0)$ & $0(0)$ & $58.7(50.0)$ & $0(0)$ & $48.0(43.9)$ & $0(0)$ & $10.9(19.3)$ & $0(0)$ \\
\hline Other & $0(0)$ & $0(0)$ & $2.2(8.6)$ & $0(0)$ & $18.4(25.4)$ & $0(0)$ & $33.3(35.3)$ & $1.1(6.2)$ & $13.0(21.1)$ & $0.6(4.3)$ \\
\hline \multicolumn{11}{|c|}{ C. minitans $\mathrm{T} 3$} \\
\hline V & $81.7(64.7)$ & $\mathrm{NC}$ & $25.4(30.3)$ & $\mathrm{NC}$ & $15.5(23.2)$ & $\mathrm{NC}$ & $8.2(16.6)$ & $\mathrm{NC}$ & $9.6(18.1)$ & $\mathrm{NC}$ \\
\hline $\mathrm{Cm}$ & $51.7(46.0)$ & $50.1(45.0)$ & $80.5(63.8)$ & $86.1(68.1)$ & $65.1(53.8)$ & $93.3(75.0)$ & $22.8(28.5)$ & $58.5(49.9)$ & $5.6(13.7)$ & $29.5(32.9)$ \\
\hline Other & $0(0)$ & $0(0)$ & $0(0)$ & $0(0)$ & $29.7(33.0)$ & $0(0)$ & $51.7(46.0)$ & $1.8(7.6)$ & $28.2(32.1)$ & $2.2(8.6)$ \\
\hline \multicolumn{11}{|c|}{$\begin{array}{l}\operatorname{LSD}(5 \%, 30 \text { d.f.) V (transformed data })=10.32^{\mathrm{e}} \\
\operatorname{LSD}(1 \%, 60 \text { d.f. }) \mathrm{Cm}\left(\text { transformed data) }=16.53^{\mathrm{f}}\right.\end{array}$} \\
\hline
\end{tabular}

${ }^{\mathrm{a}-\mathrm{g}}$ See footnotes to Table 1 for definitions. 
Table 5. Survival of Coniothyrium minitans, expressed as $\log _{10}$ colony forming units (cfu) $\mathrm{cm}^{-3}$, in four different soils amended with C. minitans isolate A69 (wild-type) or transformant T3 colonised maizemeal-perlite after 0, 1, 2, 4, 6 or 8 weeks incubation at $20{ }^{\circ} \mathrm{C}$. Mean of three replicates per treatment.

\begin{tabular}{|c|c|c|c|c|c|c|c|c|c|c|c|c|c|c|}
\hline \multirow[b]{3}{*}{ Amendment } & \multicolumn{14}{|c|}{$\log _{10} \mathrm{cfu} \mathrm{cm}^{-3}$} \\
\hline & \multicolumn{2}{|c|}{$\begin{array}{l}\text { Maizemeal-perlite } \\
\text { inoculum }\end{array}$} & \multicolumn{2}{|c|}{ Soil $0 \mathrm{wk}$} & \multicolumn{2}{|c|}{ Soil $1 \mathrm{wk}$} & \multicolumn{2}{|c|}{ Soil 2 wk } & \multicolumn{2}{|c|}{ Soil 4 wk } & \multicolumn{2}{|c|}{ Soil $6 \mathrm{wk}$} & \multicolumn{2}{|c|}{ Soil $8 \mathrm{wk}$} \\
\hline & - hyg $^{\mathrm{a}}$ & + hyg $^{\mathrm{b}}$ & - hyg & +hyg & - hyg & +hyg & - hyg & +hyg & - hyg & +hyg & - hyg & +hyg & - hyg & +hyg \\
\hline \multicolumn{15}{|l|}{ Kirton soil } \\
\hline C. minitans A69 & 7.6 & $\mathrm{NA}^{\mathrm{c}}$ & 4.5 & NA & 4.8 & NA & 4.7 & NA & 5.2 & NA & $\mathrm{NC}^{\mathrm{d}}$ & NA & 5.0 & NA \\
\hline C. minitans $\mathrm{T} 3$ & 7.7 & 7.6 & 4.7 & 4.7 & 5.0 & 5.0 & 4.9 & 5.0 & $\mathrm{NC}$ & 5.7 & 4.8 & 5.1 & 4.8 & 4.9 \\
\hline \multicolumn{15}{|l|}{ Lincoln soil } \\
\hline C. minitans A69 & 7.3 & NA & 4.2 & NA & 5.3 & NA & 5.0 & NA & 4.4 & NA & $\mathrm{NC}$ & NA & $\mathrm{NC}$ & NA \\
\hline C. minitans $\mathrm{T} 3$ & 7.4 & 7.5 & 5.4 & 5.4 & 3.9 & 3.6 & 3.8 & 5.0 & $\mathrm{NC}$ & 3.6 & $\mathrm{NC}$ & 4.0 & $\mathrm{NC}$ & 4.1 \\
\hline \multicolumn{15}{|l|}{ Rixton soil } \\
\hline C. minitans A69 & 7.6 & NA & 4.8 & NA & 4.8 & NA & 4.4 & NA & $\mathrm{NC}$ & NA & 4.5 & NA & 4.0 & NA \\
\hline C. minitans $\mathrm{T} 3$ & 7.7 & 7.6 & 4.9 & 5.0 & 4.9 & 5.0 & 4.5 & 4.9 & $\mathrm{NC}$ & 5.2 & 4.4 & 4.8 & 5.1 & 5.2 \\
\hline \multicolumn{15}{|l|}{ Wellesbourne soil } \\
\hline C. minitans A69 & 7.6 & NA & 4.3 & NA & 5.3 & NA & 5.4 & NA & $\mathrm{NC}$ & NA & $\mathrm{NC}$ & NA & 4.6 & NA \\
\hline C. minitans $\mathrm{T} 3$ & 7.7 & 7.6 & 3.7 & 4.1 & 5.4 & 5.4 & 5.3 & 5.3 & $\mathrm{NC}$ & 5.9 & 6.2 & 6.3 & 5.3 & 5.5 \\
\hline $\begin{array}{l}\operatorname{LSD}(5 \%, 38 \text { d.f. }) \\
\operatorname{LSD}(5 \%, 32 \text { d.f. }) \\
\operatorname{LSD}(5 \%, 38 \text { d.f. }) \\
\operatorname{LSD}(5 \%, 35 \text { d.f.) }\end{array}$ & $\begin{array}{l}\text { Kirton }(0 . \\
\text { incoln }(0 \\
\text { Rixton }(0 . \\
\text { Vellesbou }\end{array}$ & )$^{f}$ & & & & & & & & & & & & \\
\hline
\end{tabular}

${ }^{\text {a }}$ CDA with Triton $\mathrm{X}$ and chlortetracycline.

b CDA with hygromycin B and chlortetracycline.

c Not applicable.

d No count, too many contaminant colonies.

e LSD for comparison of colony forming units across weeks or between treatments for Kirton soil.

f For Lincoln soil.

g For Rixton soil.

h For Wellesbourne soil.

chlortetracycline and $7.5-7.6 \log _{10} \mathrm{cfu} \mathrm{cm}^{-3}$ maizemeal-perlite on CDA amended with hygromycin B (Table 5). No cfu of Coniothyrium minitans were recovered from the uninoculated control soil for any of the soils (data not shown). For all soils, cfu counts of $C$. minitans remained relatively constant throughout the experiments. In general, no differences in cfu counts of $C$. minitans were detected on the two agar types (with or without hygromycin B) for the transformants initially or after $1 \mathrm{wk}$ of incubation. For the transformant treatments, no $C$. minitans was recovered on unamended agar for all soils at $4 \mathrm{wk}$ and Lincoln soils at 6 and $8 \mathrm{wk}$ due to contaminant fungi. However, on agar containing hygromycin $\mathrm{B}$ these contaminant fungi were inhibited, enabling $C$. minitans cfu counts to be estimated. The addition of hygromycin $B$ to the agar also significantly increased recovery of $C$. minitans compared with unamended agar in both Rixton and Lincoln soil after $2 \mathrm{wk}$ and Rixton after $6 \mathrm{wk}$.

\section{DISCUSSION}

Coniothyrium minitans rapidly infected sclerotia incubated in all four soils with the pattern of sclerotial infection over time by transformant $\mathrm{T} 3$ being similar to that observed for the wild type parent A69 in each soil. However, there were clear differences in the rate of infection of sclerotia by $C$. minitans incubated in the four soils, with the fastest reduction in sclerotial viability (47\%) after 1 wk in Rixton soil, whereas for the Wellesbourne soil after $1 \mathrm{wk}$, a large proportion (over $80 \%$ ) of the sclerotia were still viable. This may relate to the low levels of indigenous fungi in the Rixton soil resulting in little competition with the $C$. minitans during the pre-infection or early infection stages. In all cases, recovery of $C$. minitans from sclerotia rapidly decreased after reaching a maximum at around 2-4 wk. Initially C. minitans is protected inside the sclerotium, but, as the sclerotia start to degrade, $C$. minitans is no longer protected and may be killed by the surface-sterilising procedure or displayed by other microbes.

Hygromycin B resistance allowed increased sensitivity in the detection of $C$. minitans from both soil and sclerotia. Using hygromycin B-amended agar, $C$. minitans was detected in soil where there was a high background of other microorganisms. This enabled the survival of $C$. minitans in soil to be quantified, with $C$. minitans seen to survive throughout the experiment. The use of the hygromycin resistant transformant also enabled the unambiguous discrimination from soil of the applied transformed $C$. minitans from the indigenous hygromycin sensitive $C$. minitans present in the Lincoln soil. In previous studies by Jones \& Whipps (2002), colony morphology based on the classification of Sandys-Winsch et al. (1993) was used to distinguish between different $C$. minitans isolates applied to soil. 
However, this can only be used when the colony morphology of the indigenous C. minitans is known and differs from that of the applied strain. Utilisation of the hygromycin B resistance marker enables the positive identification of a specific isolate regardless of colony morphology making it possible to detect and monitor the isolate in soil.

The different soils varied in their natural fungal populations, with infection by other fungi of sclerotia parasitised by $C$. minitans being high in both Wellesbourne and Lincoln soils, low in Rixton soil and intermediate in Kirton soil. Recovery of contaminating fungi from sclerotia in Kirton, Lincoln and Wellesbourne soils increased over time to a maximum level after 6 wk. For all soils, apart from Rixton, the addition of hygromycin B to the agar decreased the recovery of other fungi from sclerotia at these later harvest times, and concurrently, the detection of C. minitans increased by roughly an equivalent amount on hygromycin Bamended agar. However, when low numbers of other fungi were recovered from sclerotia as in the Rixton soil, there was no difference in the detection of C. minitans on the unamended compared with the hygromycin $\mathrm{B}$-amended agar. This indicated that the contaminating fungi were secondary colonisers of sclerotia parasitised by $C$. minitans and masked the detection of $C$. minitans on unamended agar. Other workers (McCredie \& Sivasithamparam 1985, Jones \& Stewart 2000) suggested the decline in the recovery of $C$. minitans from parasitised sclerotia over time was due to displacement of $C$. minitans by secondary colonisers. This is the first report to show that secondary colonising fungi of sclerotia infected by $C$. minitans were able to mask the detection of $C$. minitans rather than displacing the original parasite. Although hygromycin B inhibited the growth of most of the contaminating fungi, some fungi were seen to grow from infected sclerotia on hygromycin B-amended agar from all soils. Clearly, some fungi are less sensitive to hygromycin B compared with C. minitans.

There are differences in the occurrence of $C$. minitans in different soils, with only the Lincoln soil from New Zealand containing a natural population of C. minitans. Jones et al. (1999), using the same soil, also reported the occurrence of an indigenous hygromycin $\mathrm{B}$ sensitive C. minitans isolate. A large number of C. minitans isolates was isolated from both soils and sclerotia by Jones \& Stewart (2000) and there may be a difference in the natural population level of $C$. minitans in New Zealand compared with British soils. Although over $75 \%$ of sclerotia recovered after 8 wk incubation in the uninoculated Lincoln soil were infected with C. minitans, most of these sclerotia were still viable. Infection of these sclerotia by $C$. minitans may only be at the initial stages, and with increased incubation a reduction in sclerotial viability may be seen. On the other hand, the indigenous $C$. minitans isolated may be only weak sclerotial parasites, colonising only the outer surface of the sclerotia and therefore not able to kill them. Jones \& Stewart (2000) reported that C. minitans isolates recovered from New Zealand soils varied in their ability to infect and reduce sclerotial viability. The low recovery of contaminating fungi from sclerotia incubated in uninoculated Lincoln soil was also probably due to the natural $C$. minitans infection in this treatment being at an early stage and secondary colonisation of these sclerotia infected by $C$. minitans had not yet taken place. In the present study, although the indigenous C. minitans population was detected from sclerotia in the Lincoln soil, none were recovered by standard dilution plating, indicating that, at low populations, sclerotial baiting was more sensitive in detecting C. minitans from soil. In addition, the indigenous $C$. minitans population level was below the detection threshold for dilution plating. Jones \& Whipps (2002) reported that population levels of $C$. minitans below $10^{4} \mathrm{cfu} \mathrm{cm}^{-3}$ soil were close to the limit of detection, so it was not possible to quantify $C$. minitans by dilution plating at low population levels in soil. Initially, the proportion of sclerotia infected by $C$. minitans recovered from uninoculated Lincoln soil was low but, this increased over time with $75 \%$ of sclerotia infected after 8 wk. Similarly, Gerlagh \& Vos (1991) reported that enrichment of soil with $S$. sclerotiorum sclerotia led to accumulation of $C$. minitans which accelerated the loss of viability of sclerotia. This illustrates that C. minitans is a highly efficient parasite being able to infect a large proportion of sclerotia within a relatively short time from an initially low soil population.

In conclusion, the use of the hygromycin B resistance marker allowed the unambiguous discrimination of the applied transformed C. minitans isolate from the indigenous hygromycin B sensitive $C$. minitans in soil. The addition of hygromycin $B$ to agar also allowed the detection of $C$. minitans from decaying sclerotia by inhibiting secondary fungal colonisers. This study confirms that $C$. minitans is a very efficient sclerotial parasite, and is the first report to show that fungi colonising sclerotia already infected by $C$. minitans mask the detection of $C$. minitans from sclerotia rather than displacing the original parasite.

\section{ACKNOWLEDGEMENTS}

This work was funded by the New Zealand Foundation for Research, Science and Technology (contract no. LIN503), DEFRA, and BBSRC. We would like to thank Andrew Mead for statistical advice, and Naresh Magan for help with soil water potential calculations.

\section{REFERENCES}

Budge, S. P. \& Whipps, J. M. (2001) Potential for integrated control of Sclerotinia sclerotiorum in glasshouse lettuce using Coniothyrium minitans and reduced fungicide application. Phytopathology 91 : 221-227.

Challen, M. P. \& Elliott, T. J. (1986) Polypropylene straw ampoules for the storage of microorganisms in liquid nitrogen. Journal of Microbiological Methods 5: 11-23. 
Eparvier, A. \& Alabouvette, C. (1994) Use of ELISA and GUStransformed strains to study competition between pathogenic and non-pathogenic Fusarium oxysporum for root colonization. Biocontrol Science and Technology 4: 35-47.

Gerlagh, M. \& Vos, I. (1991) Enrichment of soil with sclerotia to isolate antagonists of Sclerotinia sclerotiorum. In Biotic Interactions and Soil-borne Diseases (A. B. R. Breemster, G. J. Bollen, M. Gerlagh, M. A. Ruissen, B. Schippers \& A. Tempel, eds): 165-171. Elsevier Science Publishing, Amsterdam, Netherlands.

Gerlagh, M., Goossen-van de Geijn, H. M., Fokkema, N. J. \& Vereijken, P. F. G. (1999) Long term biosanitation by application of Coniothyrium minitans on Sclerotinia sclerotiorum infected crops. Phytopathology 89: 141-147.

Green, H. \& Funck Jensen, D. (1995) A tool for monitoring Trichoderma harzianum: II. The use of a GUS transformant for ecological studies in the rhizosphere. Phytopathology 85: 1436-1440.

Green, H., Larsen, J., Olsson, P. A., Funck Jensen, D. \& Jakobsen, I. (1999) Suppression of the biocontrol agent Trichoderma harzianum by mycelium of the arbuscular mycorrhizal fungus Glomus intraradices in root-free soil. Applied and Environmental Microbiology 65: 1428-1434.

Huang, H. C. (1981) Distribution of Coniothyrium minitans in Manitoba sunflower fields. Canadian Journal of Plant Pathology $\mathbf{3}$ : 219-222.

Huang, H. C., Bremer, E., Hynes, R. K. \& Erickson, R. S. (2000) Foliar application of fungal biocontrol agents for the control of white mold in dry bean caused by Sclerotinia sclerotiorum. Biological Control 18: 270-276.

Jones, E. E., Carpenter, M., Fong, D., Goldstein, A., Thrush, A., Crowhurst, R. \& Stewart, A. (1999) Co-transformation of the sclerotial mycoparasite Coniothyrium minitans with hygromycin B resistance and $\beta$-glucuronidase markers. Mycological Research 103: 929-937.

Jones, E. E. \& Stewart, A. (2000) Selection of mycoparasites of sclerotia of Sclerotinia sclerotiorum isolated from New Zealand soils. New Zealand Journal of Crop and Horticultural Science 28: 105-114.

Jones, E. E. \& Whipps, J. M. (2002) The effect of different inoculum rates and sources of Coniothyrium minitans on control of Sclerotinia sclerotiorum disease in glasshouse lettuce. European Journal of Plant Pathology 108: 443-448.

Jones, E. E., Mead, A. \& Whipps, J. M. (2003) Evaluation of different Coniothyrium minitans inoculum sources and application rates on apothecial production and infection of Sclerotinia sclerotiorum sclerotia. Soil Biology and Biochemistry: in press.

Liljeroth, E., Jansson, H. B. \& Schäfer, W. (1993) Transformation of Bipolaris sorokiniana with the GUS gene and use for studying fungal colonization of barley roots. Phytopathology 83: 1484-1489.
McCredie, T. A. \& Sivasithamparam, K. (1985) Fungi mycoparasitic on sclerotia of Sclerotinia sclerotiorum in some Western Australian soils. Transactions of the British Mycological Society 84: 736-739.

McLaren, D. L., Huang, H. C., Kozub, G. C. \& Rimmer, S. R. (1994) Biological control of Sclerotinia wilt of sunflower with Talaromyces flavus and Coniothyrium minitans. Plant Disease 78: 231-235.

McQuilken, M. P. \& Whipps, J. M. (1995) Production, survival and evaluation of solid-substrate inocula of Coniothyrium minitans against Sclerotinia sclerotiorum. European Journal of Plant Pathology 101: 101-110.

Mylchreest, S. J. \& Wheeler, B. E. J. (1987) A method for inducing apothecia from sclerotia of Sclerotinia sclerotiorum. Plant Pathology 36: 16-20.

Oliver, R. P., Farman, M. L., Jones, J. D. G. \& Hammond-Kosack, K. E. (1993) Use of fungal transformants expressing $\beta$-glucuronidase activity to detect infection and measure hyphal biomass in infected plant tissues. Molecular Plant-Microbe Interactions 6: 521-525.

Payne, R. W. (2000) Design and analysis of experiments. In The Guide to GenStat: Part 2 -Statistics (R. W. Payne, ed.): 235-412. VSN International, Oxford.

Sandys-Winsch, C., Whipps, J. M., Gerlagh, M. \& Kruse, M. (1993) World distribution of the sclerotial mycoparasite Coniothyrium minitans. Mycological Research 97: 1175-1178.

Trutmann, P., Keane, P. J. \& Merriman, P. R. (1980) Reduction of sclerotial inoculum of Sclerotinia sclerotiorum with Coniothyrium minitans. Soil Biology and Biochemistry 12: 461-465.

Whipps, J. M., Budge, S. P. \& Ebben, M. H. (1989) Effect of Coniothyrium minitans and Trichoderma harzianum on Sclerotinia diseases in celery and lettuce in the glasshouse at a range of humidities. In Integrated Pest Management in Protected Vegetable Crops. Proceedings of the CEC-IOBC Joint Experts Meeting Cabrils, 27-29 May 1987 (R. Cavalloro \& C. Pelerents, eds): 233-243. A. A. Balkema, Rotterdam.

Whipps, J. M. \& Gerlagh, M. (1992) Biology of Coniothyrium minitans and its potential for use in disease biocontrol. Mycological Research 96: 897-907.

Whipps, J. M. \& Davies, K. G. (2000) Biocontrol of plant pathogens and nematodes by microorganisms. In Measures of Success in Biological Control (G. Gurr \& S. D. Wratten, eds): 231-269. Kluwer Academic Publishers, Dordrecht.

Williams, R. H. (1996) Dispersal of the mycoparasite Coniothyrium minitans. PhD thesis. University of Sheffield.

Corresponding Editor: S. J. Assinder 